\title{
Research on Management of Food Enterprise Technological Innovation Based on Overall Unbalanced Multiplicative Linguistic Evaluation Scale
}

\author{
Yingjun XU \\ School of Economics, Qufu Normal University, Rizhao 276826, China \\ E-mail: xuyingjun5007@163.com \\ Shijiu YIN* \\ School of Economics, Qufu Normal University, Rizhao 276826, China \\ E-mail:yinshijiu@vip.163.com \\ Mo CHEN \\ School of Economics, Qufu Normal University, Rizhao 276826, China \\ E-mail: chenmoysj@126.com \\ Yang GAO \\ School of Economics, Qufu Normal University, Rizhao 276826, China \\ E-mail:koyo521@gmail.com
}

\begin{abstract}
This paper develops a overall unbalanced multiplicative linguistic evaluation scale. Based on the developed linguistic evaluation scale and linguistic weighted averaging (LWA) operator, the technological innovation management ability of five food enterprises is evaluated. Numerical results verified the validity and utility of the scale. Meanwhile, it indicates that to improve the level of technological innovation, an enterprise should make more efforts to stimulate innovations by using the enterprises distribution system, to cultivate the leader and the staff's positive attitude to an innovation failure, to thoroughly analyze the reason of the termination of an innovation project and to strengthen the leader and the staff's innovation awareness. The overall unbalanced multiplicative linguistic evaluation scale can also be extensively applied to other fields such as project evaluation, engineering management, medical diagnosis and personnel appraisal.
\end{abstract}

Keywords multiple attribute group decision making; linguistic evaluation scale; overall unbalanced; technological innovation

\section{Introduction}

A linguistic evaluation scale is the basis of making a linguistic decision ${ }^{[1]}$. Before decision makers make linguistic decisions, they need to determine an appropriate linguistic evaluation

Received February 21, 2014, accepted April 24, 2014

*Corresponding author

Supported by National Natural Science Foundation of China (Grant No. 71203122); National Natural Science Foundation of China (Grant No. 71371107); Humanities and Social Science Research Youth Foundation of the Ministry of Education (Grant No. 13YJC790169); Natural Science Foundation of Shandong Province (Grant No. ZR2013GL002) 
scale $^{[2]}$. Bordogna et al. defined an addictive linguistic evaluation scale in which linguistic term subscripts are all nonnegative integer ${ }^{[3]}$. In the process of the integration of decision information, a situation that the integration results don't match with the element of addictive linguistic evaluation scale often occurs. In order to avoid this situation, Dai et al. defined an expanded scale on the basis of the original scale ${ }^{[4]}$. However, a situation of linguistic terms "bad" and "good" integrated into "excellent" inconsistent with the reality may appear. In order to overcome the weakness of the former scale, Xu made an improvement on addictive linguistic evaluation scale and proposed a linguistic evaluation scale with the linguistic term subscripts using zero as the symmetrical center and the number of linguistic terms is odd ${ }^{[5]}$. The subscripts of the linguistic evaluation scale proposed by Bordogna ${ }^{[3]}$ and $\mathrm{Xu}^{[5]}$ is uniform. Dai et al. ${ }^{[4]}$ thought the deviation absolute value among the adjacent linguistic term subscripts should increase with the increase of the linguistic term subscripts in practical application. Therefore, Dai et al. proposed a new additive linguistic evaluation scale, which met what's said above ${ }^{[4]}$.

Xu defined a multiplicative linguistic evaluation scale $S_{4}^{[6]}$. In $S_{4}$, the deviation absolute values between the adjacent linguistic term subscripts on the right of "1" were constant and the deviation absolute values between the adjacent linguistic term subscripts on the left of "1" increase with the increase of the linguistic term subscripts absolute values. Xu defined another multiplicative linguistic evaluation scale $S_{5}^{[7]}$. In $S_{5}$, the deviation absolute values between the adjacent linguistic term subscripts on the left of " 1 " were constant, and the deviation absolute values between the adjacent linguistic term subscripts on the right of "1" increase with the increase of the linguistic term subscripts solute values. Xu thought that $S_{4}$ and $S_{5}$ were local unbalanced linguistic evaluation scales ${ }^{[8]}$, and further research and discussion should be made on the overall unbalanced multiplicative linguistic evaluation scale. This paper constructed a overall unbalanced multiplicative linguistic evaluation scale, and made an evaluation on technological innovation management ability of five food enterprises in Shandong province by using the new linguistic evaluation scale and linguistic weighted averaging (LWA) operator. Numerical results verified the validity and utility of the new evaluation scale.

\section{An overall unbalanced multiplicative linguistic evaluation scale}

Definition 1 Construct a new linguistic evaluation scale named as OUML (Overall Unbalanced Multiplicative Linguistic, OUML) evaluation scale as follows:

$$
S=\left\{s_{\alpha} \mid \alpha=\frac{1}{n^{2}}, \frac{1}{(n-1)^{2}}, \cdots, \frac{1}{2^{2}}, 1,2^{2}, \cdots,(n-1)^{2}, n^{2}\right\}
$$

Theorem 1 Assume $S$ as formula (1) and assume the linguistic term subscripts sets on the right and left of number value 1 respectively are:

$$
\begin{gathered}
S^{+}=\left\{s_{\alpha} \mid \alpha=1,2^{2}, \cdots,(n-1)^{2}, n^{2}\right\} \\
S^{-}=\left\{s_{\alpha} \mid \alpha=\frac{1}{n^{2}}, \frac{1}{(n-1)^{2}}, \cdots, \frac{1}{2^{2}}, 1\right\}
\end{gathered}
$$

So, 1) The deviation absolute values between the adjacent linguistic term subscripts increase with the increase of the linguistic term subscripts absolute values in $\left.S^{+} ; 2\right)$ The deviation absolute values between the adjacent linguistic term subscripts increase with the increase of the linguistic term subscripts absolute values in $S^{-}$. 
Proof 1) rewrite formula (1) into $S^{+}=\left\{s_{\alpha_{i}} \mid \alpha_{i}=i^{2}, i=1,2, \cdots, \tau-1, \tau\right\}$, then, $\left|\alpha_{i+1}-\alpha_{i}\right|$ $=(i+1)^{2}-i^{2}=2 i+1, i=1,2, \cdots, \tau$. So it can be obtained that $\left|\alpha_{i+1}-\alpha_{i}\right|$ increases with the increase of $i$.

2) rewrite formula (3) into: $S^{-}=\left\{s_{\alpha_{i}} \mid \alpha_{i}=\frac{1}{(\tau-i+1)^{2}}, i=1,2, \cdots, \tau\right\}$, then,

$$
\left|\alpha_{i+1}-\alpha_{i}\right|=\left|\frac{1}{(\tau-(i+1)+1)^{2}}-\frac{1}{(\tau-i+1)^{2}}\right|=\frac{2(\tau-i)+1}{(\tau-i)^{2}(\tau-i+1)^{2}}, i=1,2, \cdots, \tau .
$$

furthermore,

$$
\frac{\mathrm{d}\left(\frac{2(\tau-i)+1}{(\tau-i)^{2}(\tau-i+1)^{2}}\right)}{\mathrm{d}(\tau-i)}=\frac{1}{(\tau-i)^{4}(\tau-i+1)^{4}}\left(-2(\tau-i)^{4}-16(\tau-i)^{3}-8(\tau-i)^{2}-2(\tau-i)\right)<0
$$

So it can be obtained that $\left|\alpha_{i+1}-\alpha_{i}\right|$ increases with the increase of $i$. The theorem is proved.

\section{An linguistic information integration operator}

On the basis of the new linguistic evaluation scale OUML and its operational rule, an LWG (Linguistic Weighted Geometric Average, LWG) operator ${ }^{[9]}$ is introduced:

Definition 2 Assume $L W G: S^{m} \rightarrow S$, if $L W G_{w}\left(s_{\alpha_{1}}, s_{\alpha_{2}}, \cdots, s_{\alpha_{n}}\right)=s_{\alpha_{1}}^{w_{1}} \otimes s_{\alpha_{2}}^{w_{2}} \otimes \cdots \otimes$ $s_{\alpha_{n}}^{w_{n}}=s_{\dot{\alpha}}$, and

$$
\dot{\bar{\alpha}}=\prod_{j=1}^{n} \alpha_{j}^{w_{j}}
$$

$w=\left(w_{1}, w_{2}, \cdots, w_{n}\right)^{T}$ is the weighting vector of linguistic data array $\left(s_{\alpha_{1}}, s_{\alpha_{2}}, \cdots, s_{\alpha_{n}}\right), w_{i} \geq$ $0, i=1,2, \cdots, n, \sum_{i=1}^{n} w_{i}=1$, the function is called as an linguistic weighted geometric averaging $(L W G)$ operator.

\section{Application in assessment on technological innovation management of food enterprise}

Under the background of global economic integration, the survival and development of an enterprise depends on it's competitive advantage ${ }^{[10]}$. Evaluating the technological innovation ability of an enterprise can help the enterprise to find problems, identify gaps, constantly improve the technological innovation ability, enhance the enterprise's economic benefit and social benefit, and remain invincible in the market competition at home and abroad ${ }^{[11]}$. Technological innovation is a basic support of ensuring food safety in quantity and quality. Promoting technological innovation of food industry is the pivotal point of the food industry restructuring. Food enterprises are the main body of technological innovation, and an evaluation on their technological innovation management system and management ability has an important significance in improving technological innovation ability of the enterprises. The present study indicated that one important method of studying technological innovation management ability is to construct a scientific, practical evaluation indicator system. Dai et al. constructed the following indicator system $^{[4]}$ : (1) the innovation system construction situation $\left(\mu_{1}\right) ;(2)$ enterprise technological innovation strategy construction and implementation $\left(\mu_{2}\right) ;(3)$ the scientificity of research and development project feasibility report $\left(\mu_{3}\right) ;(4)$ the completeness of supervision and evaluation system $\left(\mu_{4}\right) ;(5)$ the incentive degree of enterprises' distribution system on innovation $\left(\mu_{5}\right)$; 
(6) the tolerance to innovation failure $\left(\mu_{6}\right) ;(7)$ the formation and maintenance of enterprise innovation culture $\left(\mu_{7}\right)$; (8) innovation consciousness of the leaders and the staff $\left(\mu_{8}\right)$. The above indicator system can be used as a reference, but it is not difficult to find that the range of indicator (7) is too broad, and actually contains indicator (6) and indicator (8). On the reference of Oslo Manual ${ }^{[12]}$ and other literature, the paper adjusted indicator (7) into a positive attitude of the enterprises to apply for patents.

Next, the five food enterprises' technological innovation ability in Shandong province is evaluated by using the modified indicator system. First, the weights of evaluation indicator should be determined. Assume three experts from a certain university constitute judgement matrixes $R_{1}\left(r_{i j}^{1}\right), R_{2}\left(r_{i j}^{2}\right), R_{3}\left(r_{i j}^{3}\right)$ by using the Analytic Hierarchy Process (AHP) according to the relative importance of eight indicators as follows:

$$
\begin{aligned}
& R_{1}=\left(\begin{array}{cccccccc}
1.0000 & 0.5000 & 0.3333 & 0.2500 & 0.2000 & 0.1667 & 0.1429 & 0.1250 \\
2.0000 & 1.0000 & 0.5000 & 0.5000 & 0.2500 & 0.2000 & 0.1667 & 0.1429 \\
3.0000 & 2.0000 & 1.0000 & 0.5000 & 0.3333 & 0.2500 & 0.2000 & 0.1667 \\
4.0000 & 2.0000 & 2.0000 & 1.0000 & 0.5000 & 0.3333 & 0.2500 & 0.2000 \\
5.0000 & 4.0000 & 3.0000 & 2.0000 & 1.0000 & 0.5000 & 0.3333 & 0.2500 \\
6.0000 & 5.0000 & 4.0000 & 3.0000 & 2.0000 & 1.0000 & 0.5000 & 0.3333 \\
7.0000 & 6.0000 & 5.0000 & 4.0000 & 3.0000 & 2.0000 & 1.0000 & 0.5000 \\
8.0000 & 7.0000 & 6.0000 & 5.0000 & 4.0000 & 3.0000 & 2.0000 & 1.0000
\end{array}\right) \\
& R_{2}=\left(\begin{array}{llllllll}
1.0000 & 0.5000 & 0.3333 & 0.2500 & 0.1667 & 0.1667 & 0.1429 & 0.1250 \\
2.0000 & 1.0000 & 0.5000 & 0.5000 & 0.2500 & 0.1429 & 0.1667 & 0.1429 \\
3.0000 & 2.0000 & 1.0000 & 0.5000 & 0.3333 & 0.2500 & 0.2000 & 0.1667 \\
4.0000 & 2.0000 & 2.0000 & 1.0000 & 0.5000 & 0.3333 & 0.2000 & 0.2000 \\
6.0000 & 4.0000 & 3.0000 & 2.0000 & 1.0000 & 0.5000 & 0.3333 & 0.2000 \\
6.0000 & 7.0000 & 4.0000 & 3.0000 & 2.0000 & 1.0000 & 0.5000 & 0.3333 \\
7.0000 & 6.0000 & 5.0000 & 5.0000 & 3.0000 & 2.0000 & 1.0000 & 0.5000 \\
8.0000 & 7.0000 & 6.0000 & 5.0000 & 5.0000 & 3.0000 & 2.0000 & 1.0000
\end{array}\right) \\
& R_{3}=\left(\begin{array}{llllllll}
1.0000 & 0.5000 & 0.3333 & 0.2500 & 0.1667 & 0.1667 & 0.1429 & 0.1250 \\
3.0000 & 1.0000 & 0.5000 & 0.5000 & 0.2500 & 0.1429 & 0.1667 & 0.1429 \\
3.0000 & 2.0000 & 1.0000 & 0.5000 & 0.3333 & 0.2500 & 0.2000 & 0.1667 \\
4.0000 & 2.0000 & 2.0000 & 1.0000 & 0.5000 & 0.3333 & 0.2000 & 0.2000 \\
5.0000 & 4.0000 & 3.0000 & 2.0000 & 1.0000 & 0.5000 & 0.3333 & 0.2000 \\
6.0000 & 7.0000 & 4.0000 & 3.0000 & 2.0000 & 1.0000 & 0.5000 & 0.3333 \\
8.0000 & 6.0000 & 5.0000 & 5.0000 & 3.0000 & 2.0000 & 1.0000 & 0.3333 \\
8.0000 & 7.0000 & 6.0000 & 5.0000 & 5.0000 & 3.0000 & 3.0000 & 1.0000
\end{array}\right)
\end{aligned}
$$

After a calculation, three experts' judgement matrices' consistency level are 0.0274, 0.0334, 
0.0421 respectively, all below 0.1 . So, the results can be accepted without any modification. Then, use the multiplicative weighted aggregation operator as follows:

$$
r_{i j}=\prod_{k=1}^{t}\left(r_{i j}^{t}\right)^{\lambda_{t}}, i=1,2, \cdots, m, j=1,2, \cdots, n
$$

to integrate three experts' individual judgment matrix $R_{k}=\left(r_{i j}^{k}\right)_{8 \times 8}(k=1,2,3)$ into one group judgment matrix $R=\left(r_{i j}\right)_{8 \times 8}$. Among them, $\lambda_{t}(t=1,2,3)$ is the three experts' weights and assume three experts' weighting are: $\lambda_{1}=0.2, \lambda_{2}=0.5, \lambda_{3}=0.3$ based on their professional background. Integrated group judgment matrix $R=\left(r_{i j}\right)_{8 \times 8}$ is as follows:

$$
R=\left(\begin{array}{llllllll}
1.0000 & 0.5000 & 0.3333 & 0.2500 & 0.1729 & 0.1667 & 0.1429 & 0.1250 \\
2.2587 & 1.0000 & 0.5000 & 0.5000 & 0.2500 & 0.1528 & 0.1667 & 0.1429 \\
3.0000 & 2.0000 & 1.0000 & 0.5000 & 0.3333 & 0.2500 & 0.2000 & 0.1667 \\
4.0000 & 2.0000 & 2.0000 & 1.0000 & 0.5000 & 0.3333 & 0.2091 & 0.2000 \\
5.4772 & 4.0000 & 3.0000 & 2.0000 & 1.0000 & 0.5000 & 0.3333 & 0.2091 \\
6.0000 & 6.5444 & 4.0000 & 3.0000 & 2.0000 & 1.0000 & 0.5000 & 0.3333 \\
7.2861 & 6.0000 & 5.0000 & 4.7818 & 3.0000 & 2.0000 & 1.0000 & 0.4427 \\
8.0000 & 7.0000 & 6.0000 & 5.0000 & 4.7818 & 3.0000 & 2.2587 & 1.0000
\end{array}\right)
$$

In order to evaluate the similarity degree between each individual judgment matrix and group decision-making matrix, the following similarity formula ${ }^{[13]}$ is used:

$$
d\left(R_{k}, R\right)=\left(\prod_{i=1}^{m} \prod_{j=1}^{n} \frac{\min \left(r_{i j}^{k}, r_{i j}\right)}{\max \left(r_{i j}^{k}, r_{i j}\right)}\right)^{\frac{1}{m \times n}}
$$

After the calculation, the similarity degree between each individual judgment matrix and group judgment matrix are $0.9708,0.9869$ and 0.9786 respectively, so the results are acceptable. It can be concluded that group compatibility exists among three experts, which means they basically agree with each other. The eight attributes' weighting vectors can be acquired from the individual judgment matrix of expert 1: $(0.0320,0.0345,0.0480,0.0679,0.1067,0.1588,0.2323$, 0.3286 ), the eight attributes' weighting vectors from individual judgment matrix of expert 2: $(0.0223,0.0325,0.0471,0.0648,0.1043,0.1626,0.2346,0.3318)$, and the eight attributes' weighting vectors from individual judgment matrix of expert 3: (0.0211, 0.0339, 0.0468, 0.0643, $0.1011,0.1614,0.2250,0.3464)$. After integrating three experts' individual attribute weighting vectors together, the attributes' group weighting vectors are acquired: $(0.0222,0.0336,0.0473$, $0.0657,0.1040,0.1609,0.2306,0.3356)$. Therefore, the attributes which have bigger weights are incentive degree of enterprises' distribution system on innovation $\left(\mu_{5}\right)$; the tolerance degree to innovation failure $\left(\mu_{6}\right)$, positive degree of the enterprises' applying for patents $\left(\mu_{7}\right)$ and innovation consciousness of the leaders and the staff $\left(\mu_{8}\right)$. Therefore, enterprises should make efforts on these aspects if they want to improve the technological innovation ability.

Three experts make evaluation on technology innovation management conditions of five large food enterprises $x_{i}(i=1,2, \cdots, 5)$ in Shandong province by using the former eight evaluation 
indicators. The linguistic evaluation scale during the evaluation is:

$$
S=\left\{\begin{array}{l}
s_{1 / 16}=\text { expremely bad, } s_{1 / 9}=\text { very bad, } s_{1 / 4}=\text { bad, } s_{1}=\text { so so, } \\
s_{4}=\text { good, } s_{9}=\text { very good, } s_{16}=\text { expremely good }
\end{array}\right\}
$$

Three experts $d_{k}(k=1,2,3)$ gave their evaluation matrix of $x_{i}(i=1,2, \cdots, 5)$ as $A_{k}\left(a_{i j}^{(k)}\right)_{5 \times 8}(k=$ $1,2,3)$ respectively, showed as Table 1 to Table 3.

Table 1 Evaluation matrix $A_{1}$ given by expert 1

\begin{tabular}{ccccccccc} 
& $u_{1}$ & $u_{2}$ & $u_{3}$ & $u_{4}$ & $u_{5}$ & $u_{6}$ & $u_{7}$ & $u_{8}$ \\
\hline$x_{1}$ & $s_{9}$ & $s_{4}$ & $s_{16}$ & $s_{1 / 9}$ & $s_{4}$ & $s_{1}$ & $s_{1 / 9}$ & $s_{9}$ \\
$x_{2}$ & $s_{4}$ & $s_{1}$ & $s_{1 / 9}$ & $s_{1 / 4}$ & $s_{1}$ & $s_{1 / 4}$ & $s_{1 / 16}$ & $s_{1}$ \\
$x_{3}$ & $s_{1}$ & $s_{1 / 4}$ & $s_{1 / 16}$ & $s_{4}$ & $s_{1 / 4}$ & $s_{9}$ & $s_{16}$ & $s_{1 / 4}$ \\
$x_{4}$ & $s_{1 / 4}$ & $s_{9}$ & $s_{16}$ & $s_{4}$ & $s_{16}$ & $s_{4}$ & $s_{1 / 4}$ & $s_{9}$ \\
$x_{5}$ & $s_{9}$ & $s_{4}$ & $s_{1 / 4}$ & $s_{16}$ & $s_{1 / 9}$ & $s_{16}$ & $s_{4}$ & $s_{4}$ \\
\hline
\end{tabular}

Table 2 Evaluation matrix $A_{2}$ given by expert 2

\begin{tabular}{ccccccccc}
\hline & $u_{1}$ & $u_{2}$ & $u_{3}$ & $u_{4}$ & $u_{5}$ & $u_{6}$ & $u_{7}$ & $u_{8}$ \\
\hline$x_{1}$ & $s_{9}$ & $s_{4}$ & $s_{16}$ & $s_{1 / 9}$ & $s_{4}$ & $s_{1}$ & $s_{1 / 9}$ & $s_{16}$ \\
$x_{2}$ & $s_{4}$ & $s_{4}$ & $s_{1 / 9}$ & $s_{1 / 9}$ & $s_{1}$ & $s_{1 / 9}$ & $s_{1 / 16}$ & $s_{1}$ \\
$x_{3}$ & $s_{1}$ & $s_{1 / 4}$ & $s_{1 / 9}$ & $s_{4}$ & $s_{1 / 4}$ & $s_{9}$ & $s_{9}$ & $s_{1 / 9}$ \\
$x_{4}$ & $s_{1 / 16}$ & $s_{9}$ & $s_{16}$ & $s_{4}$ & $s_{9}$ & $s_{4}$ & $s_{1 / 4}$ & $s_{9}$ \\
$x_{5}$ & $s_{9}$ & $s_{4}$ & $s_{1 / 4}$ & $s_{9}$ & $s_{1 / 4}$ & $s_{9}$ & $s_{4}$ & $s_{9}$ \\
\hline
\end{tabular}

Table 3 Evaluation matrix $A_{3}$ given by expert 3

\begin{tabular}{ccccccccc}
\hline & $u_{1}$ & $u_{2}$ & $u_{3}$ & $u_{4}$ & $u_{5}$ & $u_{6}$ & $u_{7}$ & $u_{8}$ \\
\hline$x_{1}$ & $s_{4}$ & $s_{4}$ & $s_{16}$ & $s_{1 / 9}$ & $s_{9}$ & $s_{1}$ & $s_{1 / 9}$ & $s_{9}$ \\
$x_{2}$ & $s_{9}$ & $s_{9}$ & $s_{1 / 9}$ & $s_{1 / 9}$ & $s_{4}$ & $s_{1 / 9}$ & $s_{1 / 16}$ & $s_{4}$ \\
$x_{3}$ & $s_{1}$ & $s_{1 / 9}$ & $s_{1 / 16}$ & $s_{4}$ & $s_{1 / 9}$ & $s_{16}$ & $s_{9}$ & $s_{1 / 9}$ \\
$x_{4}$ & $s_{1 / 16}$ & $s_{9}$ & $s_{9}$ & $s_{4}$ & $s_{9}$ & $s_{9}$ & $s_{1 / 9}$ & $s_{9}$ \\
$x_{5}$ & $s_{9}$ & $s_{4}$ & $s_{1 / 4}$ & $s_{4}$ & $s_{1 / 4}$ & $s_{9}$ & $s_{9}$ & $s_{9}$ \\
\hline
\end{tabular}

Next, the food enterprises with the optimal technological innovation management ability are determined. First, horizontally integrate the attributes value of the $i$ th row in decision matrix $A_{k}(k=1,2,3)$ together by using the LWG operator to get each enterprise's comprehensive evaluation value $x_{i}(i=1,2,3,4,5)$ that decision makers $d_{k}(k=1,2,3)$ gave:

$$
\begin{gathered}
z_{1}^{(1)}(w)=s_{1.5794}, z_{2}^{(1)}(w)=s_{0.3582}, z_{3}^{(1)}(w)=s_{1.3456}, z_{4}^{(1)}(w)=s_{3.3015}, z_{5}^{(1)}(w)=s_{3.3085}, \\
z_{1}^{(2)}(w)=s_{1.9158}, z_{2}^{(2)}(w)=s_{0.3123}, z_{3}^{(2)}(w)=s_{0.9224}, z_{4}^{(2)}(w)=s_{3.0155}, z_{5}^{(2)}(w)=s_{4.2231}, \\
z_{1}^{(3)}(w)=s_{1.6877}, z_{2}^{(3)}(w)=s_{0.6010}, z_{3}^{(3)}(w)=s_{0.8807}, z_{4}^{(3)}(w)=s_{2.7733}, z_{5}^{(3)}(w)=s_{4.8273} .
\end{gathered}
$$

Then, integrate each enterprise's comprehensive evaluation value by using the LWG operator to get the group comprehensive evaluation value of each food enterprise $x_{i}(i=1,2,3,4,5)$ as follows:

$$
z_{1}(w)=s_{1.7744}, \quad z_{2}(w)=s_{0.3906}, \quad z_{3}(w)=s_{0.9811}, \quad z_{4}(w)=s_{2.9945}, \quad z_{5}(w)=s_{4.2016} .
$$


Last, rank the five food enterprises with $z_{i}(w)(i=1,2,3,4,5)$, and get: $x_{5}>x_{4}>x_{1}>$ $x_{3}>x_{2}$. Therefore, the fifth enterprise is the one with optimal technological innovation management ability.

\section{Conclusions}

This paper proposed a new linguistic evaluation scale that uses "1" as a symmetric center and that the number of linguistic evaluation terms is odd. The characteristic of the new evaluation scale is that the deviation among the linguistic term subscripts increases with the increase of the linguistic term subscripts absolute values, i.e., the new scale was an overall unbalanced multiplicative linguistic evaluation scale, so the related theory was perfected. The application of the new scale in technological innovation management of food enterprises indicated that the overall unbalanced multiplicative linguistic evaluation scale had a good practicability. In order to get a better overall evaluation, enterprises should attach importance to incentive degree of enterprises' distribution system on innovation, tolerance to innovation failure, emphasis on patent application, and innovation consciousness of the leaders and the staff's so as to get more policy support which is a strong base to enhance their technological innovation ability. The new linguistic evaluation scale can be applied to project evaluation, engineering management, medical diagnosis, personnel appraisal and other fields.

Acknowledgements We thank the referees for their time and comments.

\section{References}

[1] Liao X W, Li Y, Dong G M. A multi-attribute group decision-making approach dealing with linguistic assessment information. Systems Engineering — Theory \& Practice, 2006, 26(9): 90-98.

[2] Liu P D, Zhang X. Intuitionistic uncertain linguistic aggregation operations and their application to group decision making. Systems Engineering — Theory \& Practice, 2012, 32(12): 2704-2711.

[3] Bordogna G, Fedrizzi M, Passi G. A linguistic modeling of consensus in group decision making based on OWA operator. IEEE Transactions on Systems, Man, and Cybernetics, 1997, 27(1): 126-132.

[4] Dai Y Q, Xu Z S, Li Y, et al. New evaluation scale of linguistic information and its application. Chinese Journal of Management Science, 2011, 16(2): 145-149.

[5] Xu Z S. Uncertain multiple attributes decision making: Methods and applications. Qinghua University Press, Beijing, 2004.

[6] Xu Z S. EOWA and EOWG operators for aggregating linguistic labels based on linguistic preference relations. International of Uncertainty, Fuzziness and Knowledge-Based Systems, 2004, 12(6): 791-810.

[7] Xu Z S. Interactive group decision making procedure based on uncertain multiplicative linguistic preference relations. Journal of Systems Engineering and Electronics, 2010, 21(3): 408-415.

[8] Xu Z S. Linguistic decision making: Theory and methods. Science Press, Beijing, 2008.

[9] Xu Z S. On generalized induced linguistic aggregation operators. International Journal of General Systems, 2006, 35(1): 17-28.

[10] Shan H M. Application and comprehensive fuzzy evaluation of enterprise's technology innovation performance. Science Research Management, 2002, 23(6): 120-124.

[11] Hu E H. Construction and comprehensive evaluation of index system of enterprise's technological innovation capability. Science Research Management, 2001, 22(4): 79-84.

[12] Gao L C. Oslu manual: The acquisition and interpretation manual of innovation data. 3th ed. Science and Technology Literature Press, Beijing, 2011.

[13] Xu Y J, Li D. Approach to reaching consensus in multiple attribute group decision making. Control and Decision, 2011, 25(12): 1810-1814. 